\title{
ANALYZING THE MERS DISEASE CONTROL STRATEGY THROUGH AN OPTIMAL CONTROL PROBLEM
}

\author{
Dipo Aldila $^{a, *}$, Herningtyas PADMA ${ }^{a}$, KHUSNUl KHOTIMAH $^{a}$, \\ BEVINA DESJWIANDRA $^{a}$, HENGKI TASMAN ${ }^{a}$ \\ ${ }^{a}$ Department of Mathematics \\ University of Indonesia (UI), Kampus UI Depok, Depok 16424, Indonesia \\ e-mail:aldiladipo@sci.ui.ac.id
}

\begin{abstract}
A deterministic mathematical model of the Middle East respiratory syndrome (MERS) disease is introduced. Medical masks, supportive care treatment and a government campaign about the importance of medical masks will be involved in the model as time dependent variables. The problem is formulated as an optimal control one to minimize the number of infected people and keep the intervention costs as low as possible. Assuming that all control variables are constant, we find a disease free equilibrium point and an endemic equilibrium point explicitly. The existence and local stability criteria of these equilibria depend on the basic reproduction number. A sensitivity analysis of the basic reproduction number with respect to control parameters tells us that the intervention on medical mask use and the campaign about the importance of medical masks are much more effective for reducing the basic reproduction number than supportive care intervention. Numerical experiments for optimal control problems are presented for three different scenarios, i.e., a scenario of different initial conditions for the human population, a scenario of different initial basic reproduction numbers and a scenario of different budget limitations. Under budget limitations, it is much better to implement the medical mask intervention in the field, rather than give supportive care to control the spread of the MERS disease in the endemic prevention scenario. On the other hand, the medical mask intervention should be implemented partially together with supportive care to obtain the lowest number of infected people, with the lowest cost in the endemic reduction scenario.
\end{abstract}

Keywords: MERS, medical mask, supportive care, campaign, basic reproduction number, optimal control.

\section{Introduction}

MERS is a respiratory disease caused by a coronavirus or MERS-CoV, first found in Saudi Arabia in 2012 (Al-Tawfiq et al., 2012; Gautret, 2013; Zaki et al., 2012). More than 1791 cases have already been reported, with more than 640 ending in death up to 2016 (WHO, 2016). Infection with MERS can occur through direct contact between persons and/or camel to person (Reusken et al., 2013). The virus is not the same as the coronavirus that caused SARS (severe acute respiratory syndrome) in 2003. It apparently evolved by a coronavirus that existed in bats and camels. The virus can be transmitted from direct contact with infected people or through the air after sneezing (Assiri et al., 2013; Haagmans et al., 2014). The symptoms of MERS can be varied, and include fever, cough, shortness of breath, etc. Moreover, polymerase chain reaction $(\mathrm{PCR})$ tests are now available to detect

*Corresponding author
MERS-CoV in humans.

There is no specific treatment for MERS patients and no vaccine to protect people from the MERS infection, although efforts to find the vaccine are still in progress (Omrani et al., 2013; WHO, 2013). The main treatment for the MERS disease is to provide supportive care to the patients. To prevent the spreading of the MERS infection, the patient should be isolated properly and the carers should use personal protective equipment to avoid infection.

Mathematical models for some kinds of non-respiratory and respiratory diseases have been developed by many authors. The diseases include HIV-AIDS (Saha and Roy, 2017; Obaid et al., 2013), dengue (Aldila et al., 2012; Paez Chavez et al., 2017), influenza (Novkaniza et al., 2016; Xu and Ai, 2016), swine flu (Aldila et al., 2014; Pattnaik et al., 2013), tuberculosis (Gerberry, 2016; Okuonghae, 2013), 
and also MERS (Cauchemez et al., 2014; Chowell et al., 2014; Ejima et al., 2014; Malik et al., 2015; Poletto et al., 2014; Xia et al., 2015).

Cauchemez et al. (2014) estimated the incubation period and generation time from case cluster data. Chowell et al. (2014) studied a MERS-CoV transmission model with index cases and secondary cases. Ejima et al. (2014) found that the information of the incubation period upon the emergence of a novel disease and its initial growth rate is helpful in understanding the spread of the MERS. Malik et al. (2015) introduced a mathematical model for the MERS disease with a deterministic approach and accommodated vaccination to susceptible humans as well as quarantine to infected people as the intervention programs. The model was developed with a human population split into two groups (local residents and visitors). They found that the effective contact rate and the MERS-induced death rate are some of the most influential parameters to determine the spread of MERS. Poletto et al. (2014) proposed an integrative maximum likelihood to assess the MERS transmission scenario and incidence of sporadic infections. Xia et al. (2015) simulated the propagation process from the data for two different dynamical models and calculated the basic reproduction number $\mathfrak{R}_{0}$.

In this article, a mathematical model of the MERS disease with a deterministic approach will be introduced as an optimal control problem. The medical mask intervention, a health campaign about the importance of medical masks and also intervention using a supportive care treatment are introduced into the model as a time depending variable. A mathematical model will be introduced in the next section with a mathematical analysis given in the following section. In Section 4, an optimal control problem characterization will be presented and followed by numerical simulations. Some conclusions will be given in the last section.

\section{Mathematical model for optimal control of the MERS disease}

In this section, we formulate an optimal control model for the MERS disease in order to derive optimal prevention and treatment strategies with minimal implementation costs. Intervention to prevent people from contracting the MERS disease includes medical mask use and a government campaign about the importance of using medical masks to prevent people from contracting MERS. On the other hand, intervention to treat infected people is also included in the model as a supportive care intervention. Adding these interventions, it is expected that the number of healthy people can be increased at the end of the intervention period.

The control functions $u_{1}(t), u_{2}(t)$ and $u_{3}(t)$ represent time-dependent efforts of the medical mask use rate, drop-out rate and supportive care rate, respectively. Prevention efforts include how government conducts a campaign in the mass media about how important it is to use medical masks to prevent people from contracting MERS-Cov. If this campaign is a success, many people will use medical masks $\left(u_{1}\right)$, reducing the number of those who stop using them (drop out rate, $u_{2}(t)$ ). On the other hand, supportive care efforts are carried out by screening patients, and by supervising drug intake and patients' conditions. To develop the model, we

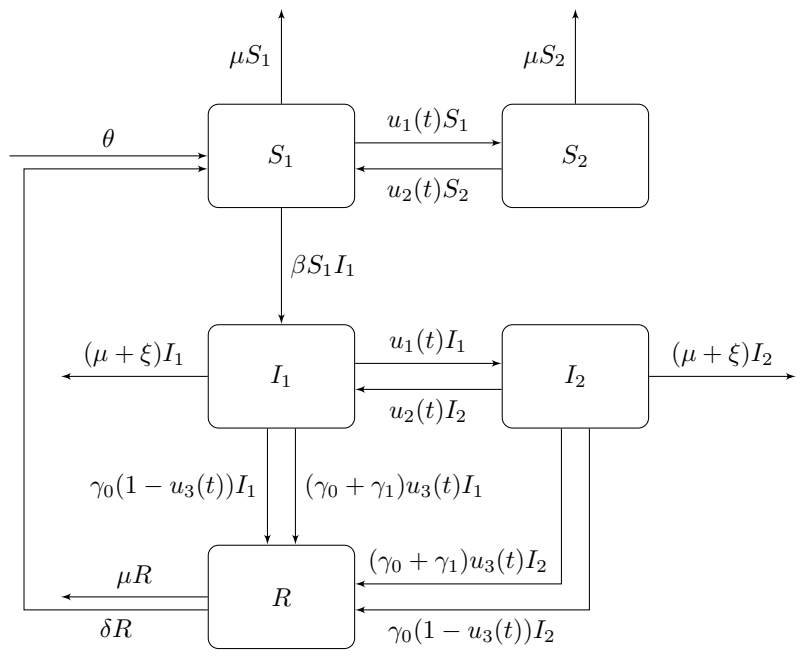

Fig. 1. Transmission diagram to construct the MERS model in the system (1).

divide the human population into five categories using $S_{1}(t), S_{2}(t), I_{1}(t), I_{2}(t)$ and $R(t)$ to describe the total number of susceptible humans, susceptible humans with medical masks, infected humans, infected humans with medical masks and recovered/temporary immune humans, respectively. Susceptible humans $S_{1}(t)$ who use medical masks will be separated into the category of susceptible humans with medical masks $\left(S_{2}(t)\right)$ and will be placed back into $S_{1}(t)$ if they stop using medical masks. The same process happens with the category of infected humans, $I_{1}(t)$ and $I_{2}(t)$. Members of the infected human $I_{1}(t)$ and $I_{2}(t)$ will get treatment which will improve their natural immune system from $\gamma_{0}$ to $\gamma_{0}+\gamma_{1}$. See Fig. 1 for the complete transmission process between all categories.

To simplify, let $S_{1}(t)=S_{1}, S_{2}(t)=S_{2}, I_{1}(t)=I_{1}$, $I_{2}(t)=I_{2}$ and $R(t)=R$. According to all assumptions explained before and the transmission diagram in Fig. 1 , the mathematical model for the spread of MERS including 
medical mask intervention and supportive care is given by

$$
\begin{aligned}
\frac{\mathrm{d} S_{1}}{\mathrm{~d} t}= & \theta-\beta S_{1} I_{1}-u_{1}(t) S_{1}+u_{2}(t) S_{2} \\
& +\delta R-\mu S_{1}, \\
\frac{\mathrm{d} S_{2}}{\mathrm{~d} t}= & u_{1}(t) S_{1}-u_{2}(t) S_{2}-\mu S_{2}, \\
\frac{\mathrm{d} I_{1}}{\mathrm{~d} t}= & \beta S_{1} I_{1}-u_{1}(t) I_{1}+u_{2}(t) I_{2} \\
& -\gamma_{0}\left(1-u_{3}(t)\right) I_{1}\left(\gamma_{0}+\gamma_{1}\right) u_{3}(t) I_{1} \\
& -(\mu+\xi) I_{1} \\
\frac{\mathrm{d} I_{2}}{\mathrm{~d} t}= & u_{1}(t) I_{1}-u_{2}(t) I_{2}-\gamma_{0}\left(1-u_{3}(t)\right) I_{2} \\
& -\left(\gamma_{0}+\gamma_{1}\right) u_{3}(t) I_{2}-(\mu+\xi) I_{2}, \\
\frac{\mathrm{d} R}{\mathrm{~d} t}= & \gamma_{0}\left(1-u_{3}(t)\right) I_{1}+\left(\gamma_{0}+\gamma_{1}\right) u_{3}(t) I_{1} \\
& +\gamma_{0}\left(1-u_{3}(t)\right) I_{2}+\left(\gamma_{0}+\gamma_{1}\right) u_{3}(t) I_{2} \\
& -\mu R-\delta R,
\end{aligned}
$$

with nonnegative initial conditions for all categories $\left(S_{1}(0) \geq 0, S_{2}(0) \geq 0, I_{1}(0) \geq 0, I_{2}(0) \geq 0, R(0) \geq 0\right)$. The total population size of humans is

$$
\begin{aligned}
N & =S_{1}(t)+S_{2}(t)+I_{1}(t)+I_{2}(t)+R(t) \\
& =\theta-\mu N-\xi\left(I_{1}(t)+I_{2}(t)\right),
\end{aligned}
$$

where $\theta, \mu$ and $\xi$ are the constant recruitment rate, natural death rate and death rate caused by MERS, respectively. Note that $u_{3}(t)$ is the rate of infected humans who received treatment. Therefore, $1-u_{3}(t)$ means the number of infected humans who did not get treatment. The rest of the functions and parameters in the model are defined as follows:

- $\theta:$ constant per capita recruitment rate [individual/day];

- $\beta$ : infection rate per capita $[($ time $\times$ day $\times$ individual $\left.)^{-1}\right]$. For example, if the infection rate of the MERS disease is 0.1 and we a total population is $N$, then $\beta$ is $0.1 / N$.

- $u_{i}(t)$ for $i=1,2,3$ described in the previous paragraph $\left[\right.$ day $\left.^{-1}\right]$.

- $\mu, \xi$ : natural death rate and death rate caused by MERS $\left[\right.$ day $\left.^{-1}\right]$.

- $\gamma_{0}, \gamma_{1}$ : natural recovery rate and effective treatments rate to increase the natural recovery rate, respectively, $\left[\right.$ day $\left.^{-1}\right]$. Thus, $\gamma_{0}\left(1-u_{3}(t)\right) I_{i}$ for $i=$ 1,2 describes the number of humans who recovered naturally, since they did not get treatment. On the other hand, $\left(\gamma_{0}+\gamma_{1}\right) u_{3}(t) I_{i}$ for $i=1,2$ describes the number of humans who recovered because of treatment intervention.
- $\delta$ : drop-out rate from $R$ to $S_{1}$ because temporary immunity vanished $\left[\right.$ day $\left.^{-1}\right]$.

For meaningful biological purposes, we assume that the recruitment rate is always positive and bounded by the upper bound $\theta_{1}$. From the system equation (1), we find that

$$
\frac{\mathrm{d} N}{\mathrm{~d} t}=\theta-\mu N-\xi\left(I_{1}+I_{2}\right) \leq \theta-\mu N .
$$

Since we assume that $N$ is constant, we obviously have $N \leq \theta / \mu$ for the initial value $N(0) \leq \theta / \mu$.

Based on the above discussion, we define

$$
\Gamma=\left\{\left(S_{1}, S_{2}, I_{1}, I_{2}, R\right) \in \mathbb{R}_{+}^{5}, 0 \leq N \leq \frac{\theta}{\mu}\right\} .
$$

In the remaining part of this article, we will restrict our human variable (further called a state variable) to this set.

Theorem 1. Let $u_{i}(t)=u_{i}$ for $i=1,2,3$. $\Gamma$ is positively invariant under the system (1).

Proof. Let

$$
\begin{aligned}
& G_{1}=-\left(\beta+u_{1}+\mu\right) \\
& G_{2}=-\left(u_{2}+\mu\right) \\
& G_{3}=-\left(u_{1}+\gamma_{0}\left(1-u_{3}\right)+\left(\gamma_{0}+\gamma_{1}\right) u_{3}+\mu+\xi\right), \\
& G_{4}=-\left(u_{2}+\gamma_{0}\left(1-u_{3}\right)+\left(\gamma_{0}+\gamma_{1}\right) u_{3}+\mu+\xi\right), \\
& G_{5}=-(\delta+\mu) .
\end{aligned}
$$

It is possible to get

$$
\frac{\mathrm{d} X_{i}}{\mathrm{~d} t} \geq G_{i} X_{i}
$$

with $X_{i}$ for $i=1-5$ replaced with $S_{1}, S_{2}, I_{1}, I_{2}, R$, respectively, and for $X_{i}(0) \geq 0$. Therefore, solutions with an initial value in $\Gamma$ remain nonnegative for all $t \geq 0$. Moreover, since

$$
\sum_{i=1}^{5} X_{i}=N
$$

from (2) we find that

$$
N \leq \frac{\theta}{\mu}
$$

Therefore, $\Gamma$ is positively invariant under the system (1).

Together with the mathematical model of MERS as described in the system (1), we consider an optimal control problem with the objective functional given by

$$
J\left(X_{i}, U_{i}\right)=\int_{0}^{T_{f}}\left(\sum_{i=1}^{5} \omega_{i} X_{i}^{2}+\sum_{j=1}^{3} \varphi_{i} u_{i}^{2}\right) \mathrm{d} t
$$


with $X_{i}$ described as in Theorem 1 . Here $\omega_{i}, i=1, \ldots, 5$ is the weight cost for the human population, whereas $\varphi_{j}, j=1,2,3$ denote weight constants for the control variable. Coefficient $\omega_{i}$ signifies a weight parameter for cost that relates to a consequence of the presence of infected people, while $\varphi_{j}$ means a weight parameter for cost that occurs because of the intervention that has to be undertaken to control the spread of MERS. Since we would like to minimize the number of infected humans and interventions efforts in Eqn. (4), we set $\omega_{i} \geq 0$ for $i=3,4$ and the rest are zero. Since higher values of $u_{1}$ and $u_{3}$ will lead to a higher intervention cost, we set $\varphi_{1}>0$ and $\varphi_{3}>0$. On the other hand, since a higher value of $u_{2}$ entails a lower effort in the campaign about medical mask importance, we set $\varphi_{2}<0$. To balance the objective functional cost, $\omega_{i}$ and $\varphi_{i}$ should satisfy

$$
\frac{\omega_{i}}{\varphi_{j}} \approx \frac{u_{j}^{2}}{X_{i}^{2}} .
$$

From the point of view of mathematics, to guarantee the existence of a solution to the optimal control problem, a convex and differentiable function for $J$ should be chosen. One alternative option is using a convex quadratic function. For this purpose, we assumed that the cost for medical masks, the drop-out rate and also treatment are in quadratic form, that is, $\varphi_{1} u_{1}^{2}$ is the cost for medical mask use, $\varphi_{2} u_{2}^{2}$ is the cost to reduce the drop-out rate from the with-medical-mask category to the without-medical-mask category, and $\varphi_{3} u_{3}^{2}$ is the cost for the supportive care effort. The cost of reducing the drop-out rate results from the government effort to implement a campaign on how important it is to wear a medical mask to reduce the spread of MERS, while the cost for treatment could come from the cost of drugs. Here $\omega_{3} x_{3}^{2}$ and $\omega_{4} x_{4}^{2}$ describe the cost of hospitalization and that resulting from an impact of the number of infected humans in the field, respectively. A higher number of infected humans will increase the cost function.

Overall, the preference of using the quadratic function as the cost function type from the biological point of view is given as follows. If the number of infected humans is still low, for example, less than 10 infected humans, then the cost to be spent to handle the infected people accounts only for the cost related to the number of infected people (almost linearly with respect to the number of infected people). On the other hand, if the number of infected people becomes larger and larger, say, than 10 , then the cost will not only relate to the treatment of the infected people, but also to improving the quality and quantity of hospital services, the cost of training health workers about MERS disease spreads, the cost related to the health campaign, etc. Therefore, the quadratic function for the cost function is one of possible options.
From the optimal control problem description in the previous paragraph, we want to seek an optimal control function $\left(u_{1}^{*}, u_{2}^{*}, u_{3}^{*}\right)$ such that

$$
\begin{aligned}
& J\left(u_{1}^{*}, u_{2}^{*}, u_{3}^{*}\right) \\
& \quad=\min \left\{J\left(u_{1}, u_{2}, u_{3}, X_{i}\right) \mid\left(u_{1}, u_{2}, u_{3}\right) \in \Psi\right\}
\end{aligned}
$$

subject to the system equation (1) and where the control set

$$
\Psi=\left(u_{1}, u_{2}, u_{3}\right) \mid u_{j}(t)
$$

is a piecewise continuous function on $\left[0, T_{f}\right], a_{j} \leq u_{j} \leq$ $b_{j}$, while $a_{j}$ and $b_{j}$ for $j=1,2,3$ are lower and upper bound control values, respectively, in $[0,1]$.

\section{Mathematical model analysis for an autonomous system}

In this section, a mathematical analysis to find the equilibrium points of the system (1) and then study their existence and stability criteria will be conducted. The basic reproduction number as an endemic criterion will also be shown in this section.

Let assume that all control variables are constant in time, $u_{j}(t)=u_{j}$ for $j=1,2,3$. Therefore, the autonomous system (1) now has the form

$$
\begin{aligned}
\frac{\mathrm{d} S_{1}}{\mathrm{~d} t}= & \theta-\beta S_{1} I_{1}-u_{1} S_{1}+u_{2} S_{2}+\delta R-\mu S_{1} \\
\frac{\mathrm{d} S_{2}}{\mathrm{~d} t}= & u_{1} S_{1}-u_{2} S_{2}-\mu S_{2} \\
\frac{\mathrm{d} I_{1}}{\mathrm{~d} t}= & \beta S_{1} I_{1}-u_{1} I_{1}+u_{2} I_{2}-\gamma_{0}\left(1-u_{3}\right) I_{1} \\
& -\left(\gamma_{0}+\gamma_{1}\right) u_{3} I_{1}-(\mu+\xi) I_{1} \\
\frac{\mathrm{d} I_{2}}{\mathrm{~d} t}= & u_{1} I_{1}-u_{2} I_{2}-\gamma_{0}\left(1-u_{3}\right) I_{2} \\
& -\left(\gamma_{0}+\gamma_{1}\right) u_{3} I_{2}-(\mu+\xi) I_{2} \\
\frac{\mathrm{d} R}{\mathrm{~d} t}= & \gamma_{0}\left(1-u_{3}\right) I_{1}+\left(\gamma_{0}+\gamma_{1}\right) u_{3} I_{1} \\
& +\gamma_{0}\left(1-u_{3}\right) I_{2}+\left(\gamma_{0}+\gamma_{1}\right) u_{3} I_{2} \\
& -\mu R-\delta R .
\end{aligned}
$$

Theorem 2. (Equilibrium points) The autonomous system (6) has two different equilibrium points, which we call disease-free equilibrium $\Omega_{1}$ point given by

$$
\begin{aligned}
& \left(S_{1}, S_{2}, I_{1}, I_{2}, R\right) \\
& =\left(\frac{\theta\left(u_{2}+\mu\right)}{\mu\left(u_{1}+u_{2}+\mu\right)}, \frac{\theta u_{1}}{\mu\left(u_{1}+u_{2}+\mu\right)}, 0,0,0\right),
\end{aligned}
$$


and an endemic equilibrium $\Omega_{2}$, given by

$$
\begin{aligned}
\left(S_{1}, S_{2}, I_{1}, I_{2}, R\right) & \\
= & \left(\frac{E\left(u_{1}+u_{2}+E\right)}{\beta\left(u_{2}+E\right)}, \frac{u_{1} E\left(u_{1}+u_{2}+E\right)}{\beta\left(\mu+u_{2}\right)\left(u_{2}+E\right)},\right. \\
& \frac{(\delta+\mu)\left(R_{1}-1\right)}{\beta G\left(u_{2}+E\right)\left(u_{1}+u_{2}+E\right)\left(\mu+u_{2}\right)}, \\
& \frac{u_{1}(\delta+\mu)\left(R_{1}-1\right)}{\beta G\left(u_{2}+E\right)\left(u_{1}+u_{2}+E\right)\left(\mu+u_{2}\right)}, \\
& \left.\frac{\left(u_{3} \gamma_{1}+\gamma_{0}\right)\left(R_{1}-1\right)}{\beta G\left(\mu+u_{2}\right)\left(u_{2}+E\right)}\right)
\end{aligned}
$$

with

$$
\begin{aligned}
& E=u_{3} \gamma_{1}+\mu+\xi+\gamma_{0}, \\
& G=\mu u_{3} \gamma_{1}+(\delta+\mu)(\mu+\xi)+\mu \gamma_{0}
\end{aligned}
$$

and

$$
\begin{aligned}
R_{1}= & \frac{\beta \theta\left(\mu+u_{2}\right)}{\mu\left(u_{3} \gamma_{1}+\mu+\xi+\gamma_{0}\right)\left(\mu+u_{1}+u_{2}\right)} \\
& \times \frac{u_{3} \gamma_{1}+\mu+u_{2}+\xi+\gamma_{0}}{u_{3} \gamma_{1}+\mu+u_{1}+u_{2}+\xi+\gamma_{0}} .
\end{aligned}
$$

Proof. Setting the right hand sides of the system (6) to zero and then solving these equations for $S_{1}, S_{2}, I_{1}, I_{2}$ and $R$ will yield the desired result.

As a biological interpretation, the disease free equilibrium point describes when all non-susceptible human categories are equal to zero. At this equilibrium point, we find that the total human population is given by $N=\theta / \mu$. On the other hand, the endemic equilibrium point $\Omega_{2}$, illustrated as a condition when all categories are positive, with a positive value criterion, is given by the next theorem.

Theorem 3. (Existence criteria) A disease-free equilibrium point $\Omega_{1}$ is always positive without any criteria, while the endemic equilibrium point will be positive if and only if $R_{1}>1$.

The next theorem will describe a criterion about of stability of the disease-free equilibrium point.

Theorem 4. ( $\Omega_{1}$ local stability criteria) A disease-free equilibrium point $\Omega_{1}$ will be locally asymptotically stable if $R_{1}<1$ and $R_{2}<1$ with $R_{1}$ given in Eqn. (8) and

$$
\begin{aligned}
R_{2}= & \frac{\beta \theta}{\mu\left(\mu+u_{1}+u_{2}\right)} \\
& \times \frac{\mu+u_{2}}{2 u_{3} \gamma_{1}+2 \mu+u_{1}+u_{2}+2 \xi+2 \gamma_{0}}
\end{aligned}
$$

Proof. Linearize the system (6) around $\Omega_{1}$ to form the Jacobian matrix

$$
J_{\Omega_{1}}=\left[\begin{array}{ccc}
J_{11} & J_{12} & J_{13} \\
J_{21} & J_{22} & J_{23}
\end{array}\right],
$$

with

$$
\begin{aligned}
& J_{11}=\left[\begin{array}{cc}
-\mu-u_{1} & u_{2} \\
u_{1} & -\mu-u_{2} \\
0 & 0
\end{array}\right] \text {, } \\
& J_{12}=\left[\begin{array}{c}
-\frac{\beta \theta\left(\mu+u_{2}\right)}{\mu\left(\mu+u_{1}+u_{2}\right)} \\
0 \\
\left(\frac{\beta \theta\left(\mu+u_{2}\right)}{\mu\left(\mu+u_{1}+u_{2}\right)}-u_{1}-\gamma_{0}\left(1-u_{3}\right)\right. \\
\left.-\left(\gamma_{0}+\gamma_{1}\right) u_{3}-\mu-\xi\right)
\end{array}\right] \text {, } \\
& J_{13}=\left[\begin{array}{cc}
0 & \delta \\
0 & 0 \\
u_{2} & 0
\end{array}\right] \text {, } \\
& J_{21}=\left[\begin{array}{ll}
0 & 0 \\
0 & 0
\end{array}\right] \text {, } \\
& J_{22}=\left[\begin{array}{c}
u_{1}, \\
\gamma_{0}\left(1-u_{3}\right)+\left(\gamma_{0}+\gamma_{1}\right) u_{3}
\end{array}\right] \text {, } \\
& J_{23}=\left[\begin{array}{c}
-u_{2}-\gamma_{0}\left(1-u_{3}\right)-\left(\gamma_{0}+\gamma_{1}\right) u_{3}-\mu-\xi \\
\gamma_{0}\left(1-u_{3}\right)+\left(\gamma_{0}+\gamma_{1}\right) u_{3}
\end{array}\right. \\
& \left.\begin{array}{c}
0 \\
-\delta-\mu
\end{array}\right]
\end{aligned}
$$

The eigenvalues of $J_{\Omega_{1}}$ are $-(\delta+\mu),-\mu,-\left(\mu+u_{1}+\right.$ $\left.u_{2}\right)$ and the other two will be given from the second order characteristic polynomial

$$
\begin{aligned}
P(\lambda) & \\
= & \left(-\mu^{2}-\mu u_{1}-\mu u_{2}\right) \lambda^{2} \\
& +\beta \theta\left(\mu+u_{2}\right)\left(u_{3} \gamma_{1}+\mu+u_{2}+\xi+\gamma_{0}\right) \\
& -\left(u_{3} \gamma_{1}+\mu+\xi+\gamma_{0}\right) \\
& \times\left(u_{3} \gamma_{1}+\mu+u_{1}+u_{2}+\xi+\gamma_{0}\right) \lambda \\
& +\beta \theta\left(\mu+u_{2}\right)-\mu\left(\mu+u_{1}+u_{2}\right) \\
& \times\left(2 u_{3} \gamma_{1}+2 \mu+u_{1}+u_{2}+2 \xi+2 \gamma_{0}\right) .
\end{aligned}
$$

The other two roots will be negative if and only if

$$
\begin{aligned}
R_{1}= & \frac{\beta \theta\left(\mu+u_{2}\right)}{\mu\left(u_{3} \gamma_{1}+\mu+\xi+\gamma_{0}\right)\left(\mu+u_{1}+u_{2}\right)} \\
& \times \frac{u_{3} \gamma_{1}+\mu+u_{2}+\xi+\gamma_{0}}{u_{3} \gamma_{1}+\mu+u_{1}+u_{2}+\xi+\gamma_{0}}<1,
\end{aligned}
$$

and unstable otherwise. 


$$
\begin{aligned}
R_{2}= & \frac{\beta \theta}{\mu\left(\mu+u_{1}+u_{2}\right)} \\
& \times \frac{\mu+u_{2}}{2 u_{3} \gamma_{1}+2 \mu+u_{1}+u_{2}+2 \xi+2 \gamma_{0}}<1 .
\end{aligned}
$$

Theorem 5. (Basic reproductive ratio) The basic reproductive ratio as the endemic criterion of the system (6) is given by

$$
\begin{aligned}
\mathfrak{R}_{0}= & \frac{\beta \theta\left(\mu+u_{2}\right)}{\mu\left(u_{3} \gamma_{1}+\mu+\xi+\gamma_{0}\right)\left(\mu+u_{1}+u_{2}\right)} \\
& \frac{u_{3} \gamma_{1}+\mu+u_{2}+\xi+\gamma_{0}}{u_{3} \gamma_{1}+\mu+u_{1}+u_{2}+\xi+\gamma_{0}} .
\end{aligned}
$$

Proof. The basic reproduction number is defined as the expected number of secondary infection cases produced by a typical infected individual during its entire period of infectiousness in a completely susceptible population (see Diekmann and Heesterbeek, 2000; Diekmann et al., 1990). Using the next generation matrix approach (Diekmann et al., 2010), we will find the basic reproduction number corresponding to (6). From the system (6), the transition matrix is given by

$$
A=\left[\begin{array}{cc}
-u_{1}-K_{1}-\mu-\xi & u_{2} \\
u_{1} & -u_{2}-K_{1}-\mu-\xi
\end{array}\right],
$$

with $K_{1}=\gamma_{0}\left(1-u_{3}\right)+\left(\gamma_{0}+\gamma_{1}\right) u_{3}$, while the transmission matrix evaluated in the disease-free equilibrium point $\Omega_{1}$ is given by

$$
B=\left[\begin{array}{cc}
\frac{\beta \theta\left(u_{2}+\mu\right)}{\mu\left(u_{1}+u_{2}+\mu\right)} & 0 \\
0 & 0
\end{array}\right] .
$$

From the work of Diekmann et al. (2010), the basic reproduction number is taken from the spectral radius of the next generation matrix. The next generation matrix of the system (6) is given by $-B A^{-1}$ in the form

$$
N G M=\left[\begin{array}{cc}
K_{2}\left(u_{2}+K_{1}+\mu+\xi\right) & K_{2} u_{2} \\
0 & 0
\end{array}\right],
$$

with

$$
K_{2}=\frac{\beta \theta\left(u_{2}+\mu\right)}{\mu\left(u_{1}+u_{2}+\mu\right) K_{3}},
$$

where $K_{3}=u_{1} K_{1}+u_{1} \mu+u_{1} \xi+K_{1} u_{2}+K_{1}^{2}+2 K_{1} \mu+$ $2 K_{1} \xi+\mu u_{2}+\mu^{2}+2 \mu \xi+\xi u_{2}+\xi^{2}$. Therefore, the spectral radius of NGM as the basic reproduction number of the system (6) is now given by

$$
\begin{aligned}
\mathcal{R}_{0}= & \frac{\beta \theta\left(\mu+u_{2}\right)}{\mu\left(u_{3} \gamma_{1}+\mu+\xi+\gamma_{0}\right)\left(\mu+u_{1}+u_{2}\right)} \\
& \times \frac{u_{3} \gamma_{1}+\mu+u_{2}+\xi+\gamma_{0}}{u_{3} \gamma_{1}+\mu+u_{1}+u_{2}+\xi+\gamma_{0}} .
\end{aligned}
$$

Furthermore, we find out that $R_{1}=\mathcal{R}_{0}$.

As a note, when $u_{i}$ in the basic reproduction number $\mathcal{R}_{0}$ is 0 , we have an initial basic reproduction number before the intervention applied, which is given by

$$
\mathcal{R}_{0}^{*}=\frac{\beta \theta}{\mu\left(\gamma_{0}+\mu+\xi\right)} .
$$

This $\mathcal{R}_{0}^{*}$ could be represented as the product of the total infection rate in an entire population $\beta \theta / \mu$ and the infection period of infected individuals $\left(\gamma_{0}+\mu+\xi\right)^{-1}$.

The next generation matrix element for the row and column describes infected humans $I_{1}$ and infected humans with medical masks $I_{2}$. Each element of the NGM matrix describes the number of secondary cases in the column space caused by one individual infected human in the row category. For example, one infected human $I_{1}$ will give a number $\mathrm{NGM}_{1,2}$ of new cases in the category of infected humans with medical masks $I_{2}$, since $I_{1}$ do not use medical masks. Therefore, the infection process still could happen in this category. In contrast, category $I_{2}$ could not produce a secondary infection in category $I_{1}$ and/or $I_{2}$, since category $I_{2}$ always uses medical masks and we also assume that medical masks could definitely reduce the spread of MERS viruses.

In Figs. 2 and 3, we give a sensitivity analysis of each of the parameters involved in the model (6) with respect to the basic reproduction number in Eqn. (11). In Fig. 2 a sensitivity analysis of $\mathcal{R}_{0}$ with respect to $u_{1}$ and $u_{2}$ as well as $u_{1}$ and $u_{3}$ is given. In Fig. 2(a), it can be seen that a larger $u_{1}$ will reduce $\mathcal{R}_{0}$ while a larger $u_{2}$ will enlarge $\mathcal{R}_{0}$. For example, choosing the medical mask use rate constant as 0.012 and the drop-out rate as 0.037 and 0.11 will give $\mathcal{R}_{0}$ values of 0.54799 and 1.092 , respectively. A larger drop-out means that the government campaign to build awareness of the importance of medical masks has been far from successful. Figure 2(b) shows that a larger number of $u_{1}$ and $u_{3}$ will reduce the number of $\mathfrak{R}_{0}$. Figure 2(b) also tells us that medical mask use as a preventive effort is much more effective than a supportive care intervention in reducing $\mathfrak{R}_{0}$.

In Fig. 3, level sets of $\mathcal{R}_{0}$ with respect to $u_{2}$ and $u_{3}$ as well as $\gamma_{1}$ and $\xi$ are given. Similarly to the previous figure interpretation, a larger value of $u_{3}$ will reduce $\mathcal{R}_{0}$ while enlarging the value of $u_{2}$ will increase $\mathcal{R}_{0}$. It is also shown that $u_{2}$ is much more sensitive to determining the value of $\mathcal{R}_{0}$ than $u_{3}$. A smaller value of $u_{2}$ is much better for reducing $\mathcal{R}_{0}$ but, as a consequence, a greater effort in the government campaign (for example, in mass media) should be made, even though this would be more expensive than the effort regarding the supportive care intervention (this result will be discussed in more detail in optimal control simulation in Section 5). Figure 3 (b) shows the level set of $\mathcal{R}_{0}$ for various values of $\gamma_{1}$ and $\xi$. It can be seen that $\gamma$ and $\xi$ are inversely proportional to $\mathcal{R}_{0}$. 


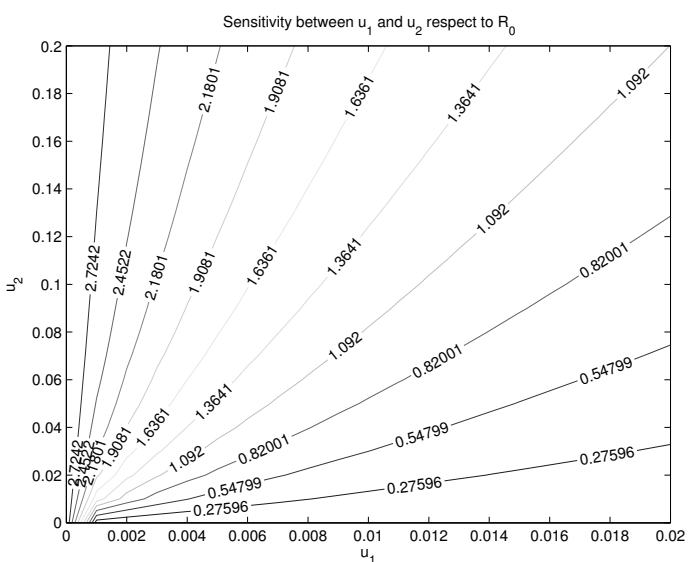

(a)

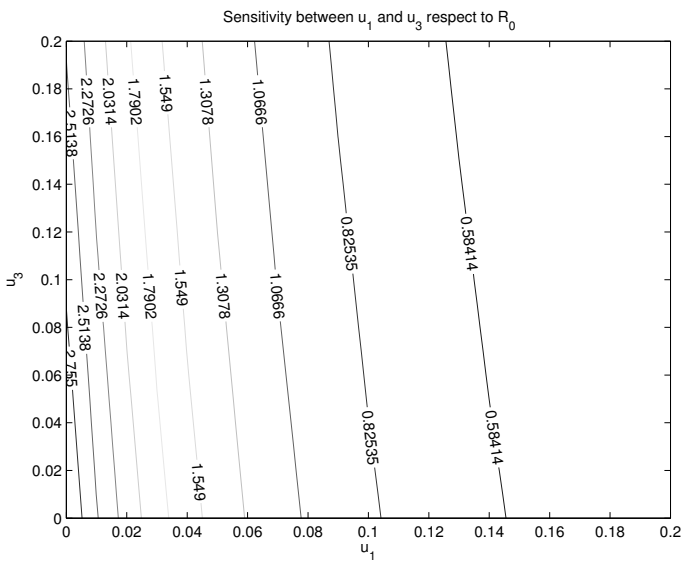

(b)

Fig. 2. Level set of $\mathcal{R}_{0}$ depends on $u_{1}$ and $u_{2}$ (a) as well as on $u_{1}$ and $u_{3}$ (b). For (a), parameter values are $\theta=$ $\frac{1000}{65 \times 365}, \beta=\frac{0.1}{1000}, \mu=\frac{1}{65 \times 365}, \xi=0, \gamma_{0}=\frac{1}{30}, \gamma_{1}=$ $\frac{1}{30}, u_{3}=0$, while those for (b) are $\theta=\frac{1000}{65 \times 365}, \beta=$ $\frac{0.1}{1000}, \mu=\frac{1}{65 \times 365}, \xi=0, \gamma_{0}=\frac{1}{30}, \gamma_{1}=\frac{1}{30}, u_{2}=0.2$.

\section{Optimal control characterization}

The optimal control characterization of the system (1) will be discussed in this section. The optimality system, which includes state equations, adjoint equations and a cost function, can be used to compute candidates for the optimal control trajectory from $t=t_{0}$ to $t=T_{f}$. Before we construct the optimal control characterization, we will use a dimensionless process with the system (1). Assuming $\xi=0$, we have that a total human population is constant $(N=\theta / \mu)$. Using the following transformation:

$$
\begin{gathered}
s_{1}=\frac{S_{1}}{N}, \quad s_{2}=\frac{S_{2}}{N}, \quad i_{1}=\frac{I_{1}}{N}, \quad i_{2}=\frac{I_{2}}{N}, \\
r=\frac{R}{N}=1-s_{1}-s_{2}-i_{1}-i_{2},
\end{gathered}
$$

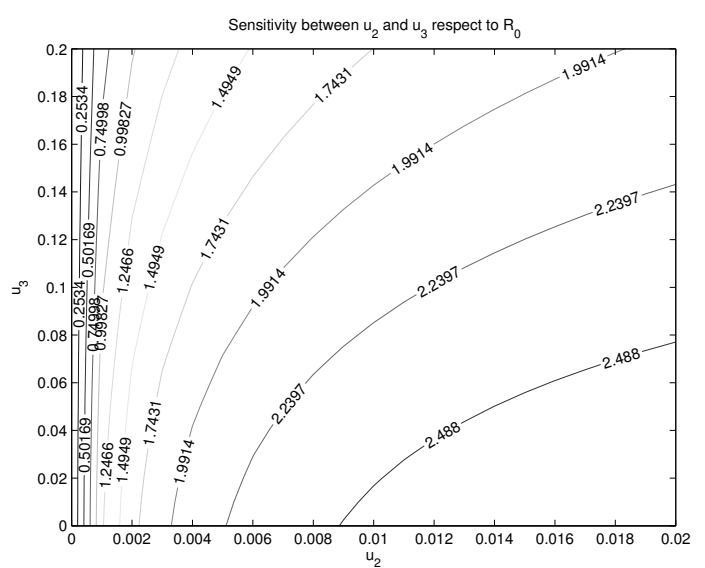

(a)

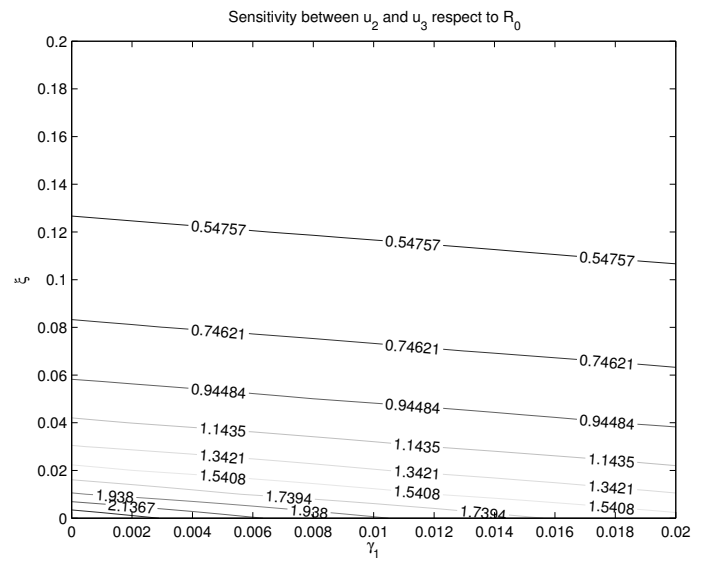

(b)

Fig. 3. Level set of $\mathcal{R}_{0}$ depends on $u_{2}$ and $u_{3}$ (a) as well as on $\gamma_{1}$ and $\xi$ (b). For (a), parameter values are $\theta=\frac{1000}{65 \times 365}, \beta=\frac{0.1}{1000}, \mu=\frac{1}{65 \times 365}, \xi=0, \gamma_{0}=$ $\frac{1}{30}, \gamma_{1}=\frac{1}{30}, u_{1}=0.01$, while for (b) the values are $\theta=\frac{1000}{65 \times 365}, \beta=\frac{0.1}{1000}, \mu=\frac{1}{65 \times 365}, \gamma_{0}=\frac{1}{30}, u_{1}=$ $0.01, u_{2}=0.1, u_{3}=0.1$.

we have that

$$
\frac{\mathrm{d} S_{1}}{\mathrm{~d} t}=\frac{\mathrm{d}\left(s_{1} N\right)}{\mathrm{d} t}
$$

becomes

$$
\begin{aligned}
\frac{\mathrm{d} s_{1} N}{\mathrm{~d} t}= & \frac{\mathrm{d} s_{1}}{\mathrm{~d} t} N \\
= & \mu N-\beta s_{1} N i_{1} N-u_{1} s_{1} N+u_{2} s_{2} N \\
& +\delta r N-\mu s_{1} N .
\end{aligned}
$$

Dividing each side by $N$, we get

$$
\frac{\mathrm{d} s_{1}}{\mathrm{~d} t}=\mu-\beta s_{1} i_{1} N-u_{1} s_{1}+u_{2} s_{2}+\delta r-\mu s_{1} .
$$

Repeating this process with the other equations in the system (1), we reduce our five-dimensional system to the 
four-dimensional system given as

$$
\begin{aligned}
\frac{\mathrm{d} s_{1}}{\mathrm{~d} t}= & \mu-N \beta s_{1} i_{1}-u_{1}(t) s_{1}+u_{2}(t) s_{2} \\
& +\delta\left(1-s_{1}-s_{2}-i_{1}-i_{2}\right)-\mu s_{1} \\
\frac{\mathrm{d} s_{2}}{\mathrm{~d} t}= & u_{1}(t) s_{1}-u_{2}(t) s_{2}-\mu s_{2} \\
\frac{\mathrm{d} i_{1}}{\mathrm{~d} t}= & N \beta s_{1} i_{1}-u_{1}(t) i_{1}+u_{2}(t) i_{2} \\
& -\gamma_{0}\left(1-u_{3}(t)\right) i_{1} \\
\frac{\mathrm{d} i_{2}}{\mathrm{~d} t}= & u_{1}(t) i_{1}-\gamma_{0}\left(1-u_{3}(t)\right) i_{2} \\
& -u_{2}(t) i_{2}-\left(\gamma_{0}+\gamma_{1}\right) u_{3}(t) i_{2} .
\end{aligned}
$$

Note that $s_{1}, s_{2}, i_{1}, i_{2}$ and $r$ represent the proportion of each compartment with respect to the total of human population $N$.

In the next step we define the Lagrangian. Let $x_{i} \in$ $\Gamma_{2}$ for $i=1,2,3,4$, represent $s_{1}, s_{2}, i_{1}, i_{2}$, respectively, as state variables and $U_{i}=\left(u_{1}, u_{2}, u_{3}\right) \in \Psi$ with $\Gamma_{2} \in[0,1]$, and $\Psi$ defined in (5). The Lagrangian for our problem consists of the integrand of the objective functional given by

$$
J\left(x_{i}, u_{i}\right)=\int_{0}^{T_{f}}\left(\sum_{i=1}^{4} \omega_{i} x_{i}^{2}+\sum_{j=1}^{3} \varphi_{i} u_{i}^{2}\right) \mathrm{d} t
$$

and the inner product of the right hand sides of the state equations (18) and the adjoint variables $\Lambda=$ $\left(\lambda_{1}, \lambda_{2}, \lambda_{3}, \lambda_{4}\right)$. Now, let us define the Lagrangian $\mathcal{L}\left(X_{i}, U_{i}, \Lambda\right)$ as below:

$$
\begin{aligned}
\mathcal{L}= & \sum_{i=1}^{4} \omega_{i} x_{i}^{2}+\sum_{j=1}^{3} \varphi_{i} u_{i}^{2} \\
& +\lambda_{1}\left(\mu-N \beta s_{1} i_{1}-u_{1}(t) s_{1}+u_{2}(t) s_{2}\right. \\
& \left.+\delta\left(1-s_{1}-s_{2}-i_{1}-i_{2}\right)-\mu s_{1}\right) \\
& +\lambda_{2}\left(u_{1}(t) s_{1}-u_{2}(t) s_{2}-\mu s_{2}\right) \\
& +\lambda_{3}\left(N \beta s_{1} i_{1}-\left(u_{1}(t)+\mu+\xi\right) i_{1}+u_{2}(t) i_{2}\right. \\
& \left.-\gamma_{0}\left(1-u_{3}(t)\right) i_{1}-\left(\gamma_{0}+\gamma_{1}\right) u_{3}(t) i_{1}\right) \\
& +\lambda_{4}\left(u_{1}(t) i_{1}-u_{2}(t) i_{2}\right. \\
& -\gamma_{0}\left(1-u_{3}(t)\right) i_{2}-\left(\gamma_{0}+\gamma_{1}\right) u_{3}(t) i_{2} \\
& \left.-(\mu+\xi) i_{2}\right) .
\end{aligned}
$$

Theorem 6. Given an optimal control function $\left(\bar{u}_{1}, \bar{u}_{2}, \bar{u}_{3}\right)$ and trajectory solutions for $s_{1}, s_{2}, i_{1}, i_{2}$ of the corresponding system (18), there exist adjoint variables
$\Lambda_{1}, \ldots, \Lambda_{4}$ satisfying

$$
\begin{aligned}
\dot{\lambda_{1}}= & \lambda_{1} N \beta i_{1}+\left(\lambda_{1}-\lambda_{2}\right) u_{1}+\lambda_{1} \mu \\
& -\lambda_{3} N \beta i_{1}, \\
\dot{\lambda_{2}}= & \left(\lambda_{2}-\lambda_{1}\right) u_{2}+\lambda_{1} \delta+\lambda_{2} \mu, \\
\dot{\lambda_{3}}= & -2 \omega_{3} i_{1}-\lambda_{1}\left(-N \beta s_{1}-\delta\right) \\
& +\lambda_{3} \gamma_{0}\left(1-u_{3}\right)+\lambda_{3}\left(\gamma_{0}+\gamma_{1}\right) u_{3} \\
& -\lambda_{3} N \beta s_{1}+\lambda_{3} \mu+\left(\lambda_{3}-\lambda_{4}\right) u_{1}, \\
\dot{\lambda_{4}}= & -2 \omega_{4} i_{2}+\lambda_{4} \mu+\lambda_{4} \xi \\
& +\lambda_{4} \gamma_{0}\left(1-u_{3}\right)+\lambda_{4}\left(\gamma_{0}+\gamma_{1}\right) u_{3} \\
& +\lambda_{1} \delta\left(\lambda_{4}-\lambda_{3}\right) u_{2},
\end{aligned}
$$

with the terminal condition

$$
\lambda_{i}\left(T_{f}\right)=0 \text { for } i=1,2,3,4 .
$$

Furthermore, $\bar{u}_{1}, \bar{u}_{2}$ and $\bar{u}_{3}$ are represented by

$$
\begin{aligned}
\bar{u}_{1}= & \max \left(a_{1}, \min \left(b_{1},\right.\right. \\
& \left.\left.\frac{s_{1}\left(\lambda_{1}-\lambda_{2}\right)+i_{1}\left(\lambda_{3}-\lambda_{4}\right)}{2 \varphi_{1}}\right)\right), \\
\bar{u}_{2}= & \max \left(a_{2}, \min \left(b_{2},\right.\right. \\
& \left.\left.\frac{s_{2}\left(\lambda_{2}-\lambda_{1}\right)+i_{2}\left(\lambda_{4}-\lambda_{3}\right)}{2 \varphi_{2}}\right)\right), \\
\bar{u}_{3}= & \max \left(a_{3}, \min \left(b_{3},\right.\right. \\
& \left.\left.\frac{\gamma_{1} i_{1}\left(\lambda_{3}-\lambda_{5}\right)+\gamma_{1} i_{2}\left(\lambda_{4}-\lambda_{5}\right)}{2 \varphi_{3}}\right)\right),
\end{aligned}
$$

with $a_{i}$ and $b_{i}$ for $i=1,2,3$ being the lower and the upper bounds for each control variable, respectively.

Proof. First, we differentiate the Lagrangian (20) with respect to each state variable and then get the equations for as the adjoint variables

$$
\begin{aligned}
& \dot{\lambda_{1}}=-\frac{\partial L}{\partial s_{1}}, \quad \dot{\lambda_{2}}=-\frac{\partial L}{\partial s_{2}}, \\
& \dot{\lambda_{3}}=-\frac{\partial L}{\partial i_{1}}, \quad \dot{\lambda_{4}}=-\frac{\partial L}{\partial i_{2}}
\end{aligned}
$$

with the terminal conditions $\lambda_{i}\left(T_{f}\right)=0$ for $i=1,2,3,4$.

To obtain the optimality conditions (23), we will also differentiate the Lagrangian (20) with respect to $u_{1}, u_{2}$ and $u_{3}$, which gives

$$
\begin{aligned}
\frac{\partial H}{\partial u_{1}}= & 2 \varphi_{1} u_{1}-\lambda_{1} s_{1}+\lambda_{2} s_{1}-\lambda_{3} i_{1}+\lambda_{4} i_{1}=0, \\
\frac{\partial H}{\partial u_{2}}= & 2 \varphi_{2} u_{2}+\lambda_{1} s_{2}-\lambda_{2} s_{2}+\lambda_{3} i_{2}-\lambda_{4} i_{2}=0, \\
\frac{\partial H}{\partial u_{3}}= & 2 \varphi_{3} u_{3}+\lambda_{3}\left(\gamma_{0} i_{1}+\left(\gamma_{o}+\gamma_{1}\right) i_{1}\right) \\
& +\lambda_{4}\left(\gamma_{0} i_{2}-\left(\gamma_{0}+\gamma_{1}\right) i_{2}\right)=0,
\end{aligned}
$$


and set these equations equal to zero. Solving them with respect to each control variable, we obtain

$$
\begin{aligned}
\overline{u_{1}}(t)= & \frac{1}{2 \varphi_{1}}\left(\lambda_{1} s_{1}-\lambda_{2} s_{1}+\lambda_{3} i_{1}-\lambda_{4} i_{1}\right), \\
\overline{u_{2}}(t)= & \frac{1}{2 \varphi_{2}}\left(-\lambda_{1} s_{2}+\lambda_{2} s_{2}-\lambda_{3} i_{2}+\lambda_{4} i_{2}\right), \\
\overline{u_{3}}(t)= & \frac{1}{2 \varphi_{3}}\left(\lambda_{3}\left(\gamma_{0} i_{1}-\left(\gamma_{o}+\gamma_{1}\right) i_{1}\right)\right. \\
& \left.-\lambda_{4}\left(\gamma_{0} i_{2}-\left(\gamma_{0}+\gamma_{1}\right) i_{2}\right)\right) .
\end{aligned}
$$

To determine an acceptable control variables value based on the needs and ability in field applications (lower and upper bounds), the optimal control variable now is

$$
\begin{aligned}
\overline{u_{1}}= & \max \left(a_{1}, \min \left(b_{1},\right.\right. \\
& \left.\left.\frac{s_{1}\left(\lambda_{1}-\lambda_{2}\right)+i_{1}\left(\lambda_{3}-\lambda_{4}\right)}{2 \varphi_{1}}\right)\right), \\
\overline{u_{2}}= & \max \left(a_{2}, \min \left(b_{2},\right.\right. \\
& \left.\left.\frac{s_{2}\left(\lambda_{2}-\lambda_{1}\right)+i_{2}\left(\lambda_{4}-\lambda_{3}\right)}{2 \varphi_{2}}\right)\right) \\
\overline{u_{3}}= & \max \left(a_{3}, \min \left(b_{3},\right.\right. \\
& \left.\left.\frac{\gamma_{1} i_{1}\left(\lambda_{3}-\lambda_{5}\right)+\gamma_{1} i_{2}\left(\lambda_{4}-\lambda_{5}\right)}{2 \varphi_{3}}\right)\right),
\end{aligned}
$$

with $a_{i}$ and $b_{i}$ for $i=1,2,3$ being the lower and upper bounds for each control variable, respectively.

Now, we point out that the optimality system consists of the state system (18) with the initial condition $\left(s_{1}(0), s_{2}(0), i_{1}(0), i_{2}(0)\right)$, the adjoint system (21) with the terminal condition $\lambda_{i}\left(T_{f}\right)=0$, and the optimality conditions 23. Any optimal control trajectories must satisfy this optimality system. A numerical simulation for this situation will be given in the next section.

\section{Numerical simulation results}

In this section, numerical simulations of the MERS disease spread are reported. We discuss results for the autonomous model (6) and the optimal control problem for various scenarios.

5.1. Simulation of an autonomous model. In this section, various scenarios of constant parameters and initial conditions are presented to show the possible dynamics of the system (6). The first simulation is presented for two different cases: before $\left(\mathcal{R}_{0}=2.996\right)$ and after intervention $\left(\mathcal{R}_{0}=0.737\right)$, as shown in Fig. 4 (cf. Table 1 for details about various scenarios for the value of $u_{i}$ ). It can be seen that the intervention of all control variables $\left(u_{i}\right)$ partially succeeds in reducing the basic reproduction number and makes the trajectory move from an endemic equilibrium to a disease-free equilibrium

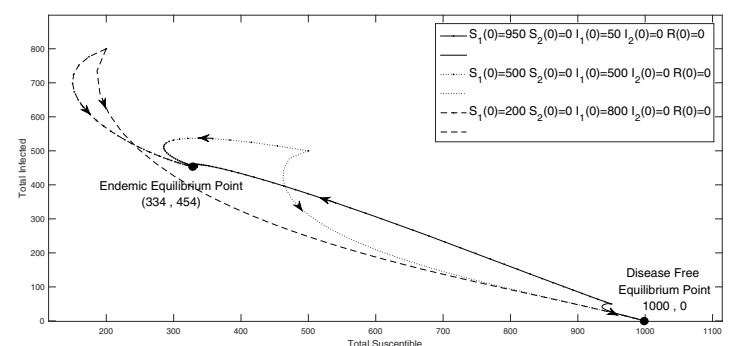

Fig. 4. Trajectory solutions for the total of susceptible humans $\left(S_{1}(t)+S_{2}(t)\right)$ and total infected humans $\left(I_{1}(t)+I_{2}(t)\right)$ without control (dotted line) and with control (dashed line). We use a constant control value $\left(u_{1}=0.3, u_{2}=0.5, u_{3}=0.65\right)$ while the other parameters remain the same for both scenarios.

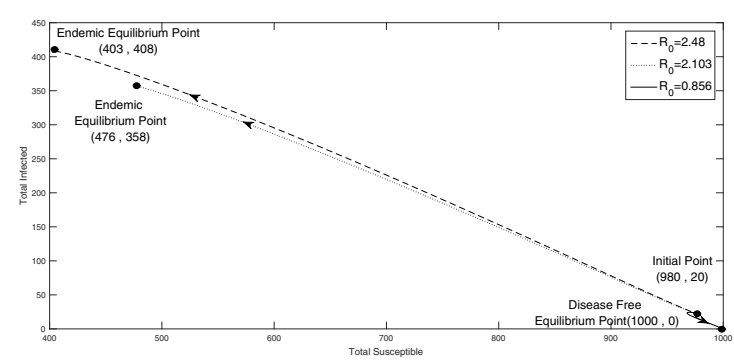

Fig. 5. Trajectory solutions for total susceptible $\left(S_{1}(t)+S_{2}(t)\right)$ and total infected $\left(I_{1}(t)+I_{2}(t)\right)$ humans with various values of $u_{2}(1,0.5,0.1)$ and $u_{1}$ that remain constant in 0.1 . All other parameters remain the same for the three scenarios.

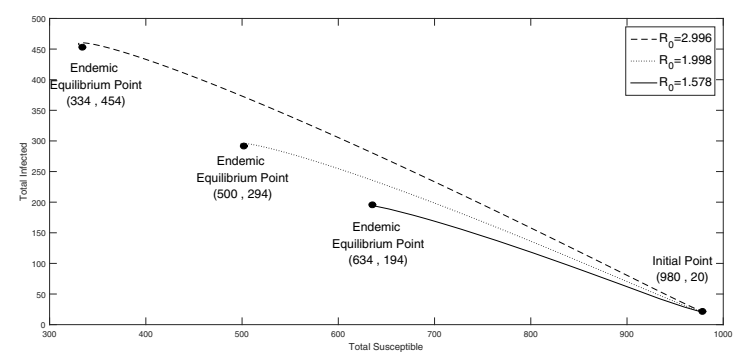

Fig. 6. Trajectory solutions for the total of susceptible humans $\left(S_{1}(t)+S_{2}(t)\right)$ and total infected $\left(I_{1}(t)+I_{2}(t)\right)$ humans with various values of $u_{3}(0,0.5,0.9)$ as well as $u_{1}$ and $u_{2}$ that remain constant in at zero. All other parameters remain the same for the three scenarios.

point. We use some different initial conditions to give a better simulation result. From Theorem 1, it can be seen that in Fig. 4 the trajectories remain positive all the time and tend to two different equilibrium points as already stated in Theorem 2. It is clear from Theorem 4, that disease-free equilibrium points $\Omega_{1}$ are locally stable for $\mathcal{R}_{0}<1$. The trajectories tend to a disease-free equilibrium point for $\mathcal{R}_{0}<1$ and to an endemic 
equilibrium point for $\mathcal{R}_{0}>1$.

The next simulation is given in Figs. 5 and 6 to represent two different intervention scenarios, i.e., with the medical mask intervention only $\left(u_{1}>0, u_{2}>\right.$ $\left.0, u_{3}=0\right)$ and with supportive care only $\left(u_{1}=0, u_{2}=\right.$ $\left.0, u_{3}>0\right)$, respectively. All parameter values are the same as in the previous simulation in Fig. 4, except for $u_{i}$ for $i=1,2,3$.

Figure 5] shows the effect of medical mask intervention to reduce the basic reproduction number and the number of infected people. We can see that we need a specific level of $u_{2}$ to reduce the basic reproduction number to less than 1 so that we can move the dynamic from the endemic equilibrium point to the disease-free equilibrium point. This confirm our result for the level set of the basic reproduction number with respect to $u_{1}$ and $u_{2}$ in Fig. 2(a) that increasing $u_{1}$ and reducing $u_{2}$ will reduce the basic reproduction number to less than one, which will make the disease free equilibrium point stable.

In contrast to Fig. 5, Fig. 6 shows the effect of the supportive care intervention only to reduce the magnitude of the basic reproduction number. As shown in Table 1, with only the intervention of $u_{3}$, the basic reproduction number cannot be reduced to less than 1 since with $u_{3}=1$ we can only reach the magnitude of the basic reproduction number of 1.578 as the minimum possibility. However, we can reduce the total number of infected people to a lower level with this scenario, even though not until no more infected people exist in the environment, as shown in Fig. 6

5.2. Simulation of the optimal control problem. The optimality system is a two-point boundary value one, since we know the initial condition for the state variable $\left(x_{i}(0)\right)$ in Eqn. (18) and the final condition for the adjoint system $\left(\lambda_{i}\left(T_{f}\right)\right)$ in Eqn. (21). In this optimal control problem, we use an iterative method based on the gradient descent algorithm, given in Algorithm 1.

To simulate the optimal control problem of the system described in Theorem 6, we will give some different scenarios.

\subsubsection{Simulation for different initial conditions.} This section will describe how control variables adapt to different situations for the initial value of each category $\left(x_{i}(0)\right)$, Let us call it an endemic prevention scenario (when the initial number of infected people is at a low level), and an endemic reduction scenario (when the initial number of infected people is at a high level). Table 2 details the initial condition.
Algorithm 1. Gradient descent.

$\overline{\text { Step 1. Fix a constant initial guess for } u_{1}, u_{2}, u_{3} \text { for } t \in}$ $\left[0, T_{f}\right]$.

Step 2. Solve the state equation (18) with respect to the initial value of the control variable and the initial condition of the state variable forward in time.

Step 3. Calculate the cost function (19).

Step 4. Solve the adjoint system (21) with final time $\lambda_{i}\left(T_{f}\right)=0$ for $i=1,2,3,4$ backward in time.

Step 5. Solve the gradient equation to get the descent vector $u_{i}^{*}$ supplemented with $x_{i}$ and $\lambda_{i}$ from Steps 2 and 4. Use the actual values $\left\{u_{i}^{*}\right\}$ at particular times to update the values of $u_{i}$ in Step 1 .

Step 6. Go to Step 2 and iterate until convergence criteria are achieved.

The first simulation is given for the endemic prevention scenario. The trajectories of total susceptible and infected people are given in Fig. 7 as a result of control intervention that varied in time, shown in Fig. 8. Table 3 describes the effect of interventions on
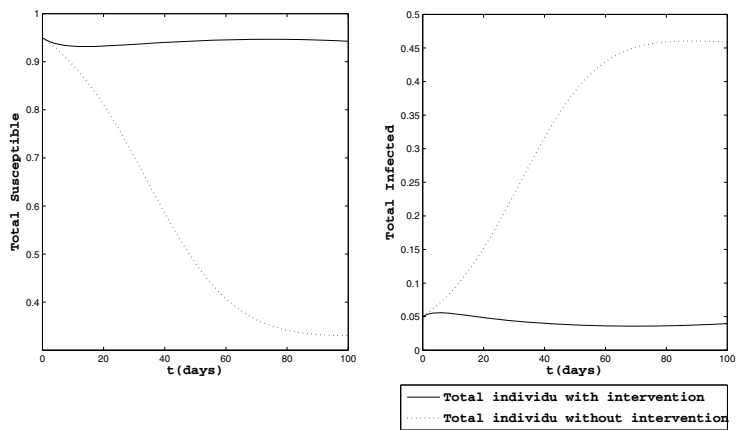

Fig. 7. Dynamics of total susceptible (left panel) and infected (right panel) humans with and without intervention strategies in the endemic prevention scenario.
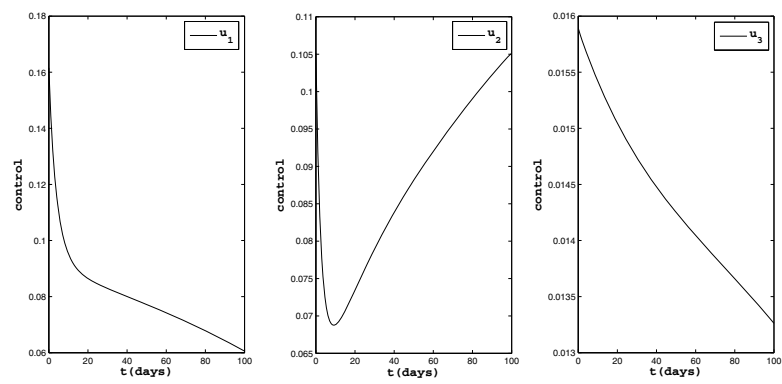

Fig. 8. Dynamics of control intervention for the endemic prevention scenario. 
Table 1. Equilibrium points of the system (6) for Figs. 4.6

\begin{tabular}{|c|c|c|ccccc|}
\hline Figure & $u_{i}$ & $\mathcal{R}_{0}$ & \multicolumn{5}{c|}{ Categories } \\
& & & $S_{1}$ & $S_{2}$ & $I_{1}$ & $I_{2}$ & $R$ \\
\hline \hline Fig.[4] & $u_{1}=0, u_{2}=0, u_{3}=0$ & 2.996 & 333.75 & 0 & 454.34 & 0 & 211.91 \\
& $u_{1}=0.3, u_{2}=0.5, u_{3}=0.65$ & 0.737 & 625.02 & 374.98 & 0 & 0 & 0 \\
\hline \multirow{3}{*}{ Fig.5] } & $u_{1}=0.1, u_{2}=1, u_{3}=0$ & 2.484 & 366.05 & 36.6 & 371.41 & 35.49 & 189.98 \\
& $u_{1}=0.1, u_{2}=0.5, u_{3}=0$ & 2.103 & 396.29 & 79.25 & 301.15 & 56.43 & 166.79 \\
& $u_{1}=0.1, u_{2}=0.1, u_{3}=0$ & 0.856 & 500.1 & 499.9 & 0 & 0 & 0 \\
\hline \multirow{3}{*}{ Fig.66 } & $u_{1}=0, u_{2}=0, u_{3}=0$ & 2.996 & 333.75 & 0 & 454.34 & 0 & 211.9 \\
& $u_{1}=0, u_{2}=0, u_{3}=0.5$ & 1.998 & 500.42 & 0 & 293.94 & 0 & 205.63 \\
& $u_{1}=0, u_{2}=0, u_{3}=0.9$ & 1.578 & 633.75 & 0 & 194.17 & 0 & 172.07 \\
\hline
\end{tabular}

Table 2. Initial condition of each category for the endemic prevention and reduction scenario.

\begin{tabular}{|c|ccccc|}
\hline Scenario & \multicolumn{5}{|c|}{ Compartments } \\
& $s_{1}(0)$ & $s_{2}(0)$ & $i_{1}(0)$ & $i_{2}(0)$ & $r(0)$ \\
\hline \hline Endemic prevention & 0.95 & 0 & 0.05 & 0 & 0 \\
Endemic reduction & 0.7 & 0 & 0.3 & 0 & 0 \\
\hline
\end{tabular}

increasing/decreasing the total susceptible and infected categories.

The second simulation is given for the endemic reduction scenario, when the initial infected population
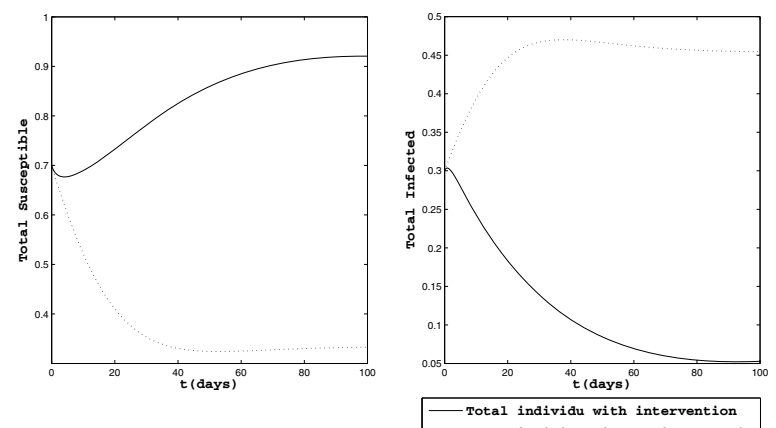

Total individu without intervention

Fig. 9. Dynamics of total susceptible (left panel) and infected (right panel) populations with and without intervention for the endemic reduction scenario.
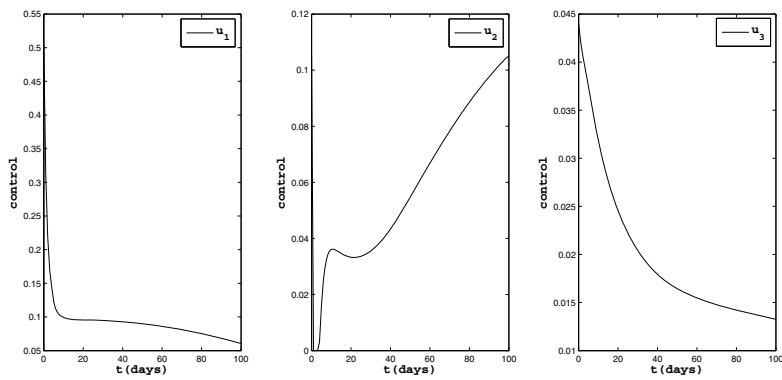

Fig. 10. Dynamics of control intervention for the endemic reduction scenario. was higher than in the endemic prevention scenario. The dynamics of the control variables in Fig. 10 will have the effect of increasing the susceptible population and decreasing the infected population, as shown in Fig. 9 .

From both the simulations above, intervention using all control variables succeeded in increasing the total susceptible population to more than $90 \%$ and suppressing the infected population to less than $6 \%$. Nonetheless, different behavior was apparent in the control simulation. It can be seen that a more intense intervention should be applied in the endemic reduction scenario, rather than in the endemic prevention scenario. The magnitude of the cost function for these scenarios is 0.0288 and 0.2585 for the endemic prevention and endemic reduction scenarios, respectively. It is shown that, from $t=0$, intervention using a medical mask $\left(u_{1}\right)$ should occur at a high rate (about $50 \%$ of the total population should use medical masks) in the endemic reduction scenario. Compared with the endemic prevention scenario, control using medical masks only needs the compliance of about $16 \%$ of the total population who should use medical masks at the initial time. To guarantee the success of medical mask use interventions, the government campaign about the importance of medical mask use should be intensified. An extreme result is shown in the endemic reduction scenario, since $u_{2}(t)$ reached zero early in the simulation. This means that in the endemic reduction scenario, no individual in $S_{2}$ should be allowed to take off their medical masks for a certain period during the simulation.

5.2.2. Simulation with different $\mathcal{R}_{0}^{*}$ scenarios. In this subsection, we report two different scenarios for each simulation. The first simulation is for $\mathcal{R}_{0}<1$, which describes a healthy environment where with or without the intervention of control variables the dynamics 
Table 3. Numerical result for control intervention in endemic prevention and endemic reduction scenarios.

\begin{tabular}{|c|c|c|c|c|}
\hline Compartment & $\begin{array}{l}\text { Endemic preve } \\
\text { without control }\end{array}$ & $\begin{array}{l}\text { on }(t=100) \\
\text { with control }\end{array}$ & $\begin{array}{l}\text { Endemic reduc } \\
\text { without control }\end{array}$ & $\begin{array}{l}\text { n }(t=100) \\
\text { with control }\end{array}$ \\
\hline$s_{1}+s_{2}$ & 0.3302 & 0.9425 & 0.3302 & 0.9205 \\
\hline$i_{1}+i_{2}$ & 0.4590 & 0.0397 & 0.4590 & 0.0529 \\
\hline
\end{tabular}

of the system will tend to move towards a disease-free equilibrium point. In this case, intervention is needed to accelerate the disappearance of MERS in the population. On the other hand, the second simulation is for $\mathfrak{R}_{0}>$ 1 , which describes an unhealthy environment, i.e., the dynamics of the system will tend to move towards an endemic equilibrium point if interventions are not present to the population. For this purpose, we set all parameters for each simulation to remain the same except for $\beta$, i.e., $\beta=0.000028$ for $\mathcal{R}_{0}=0.84$ and $\beta=0.000081$ for $\mathcal{R}_{0}=2.43$.

As already stated in Theorem 4, the disease-free equilibrium will be locally asymptotically stable if $\mathcal{R}_{0}<$ 1 and unstable otherwise. With the intervention of all control variables simultaneously, as shown in Fig. 12, we
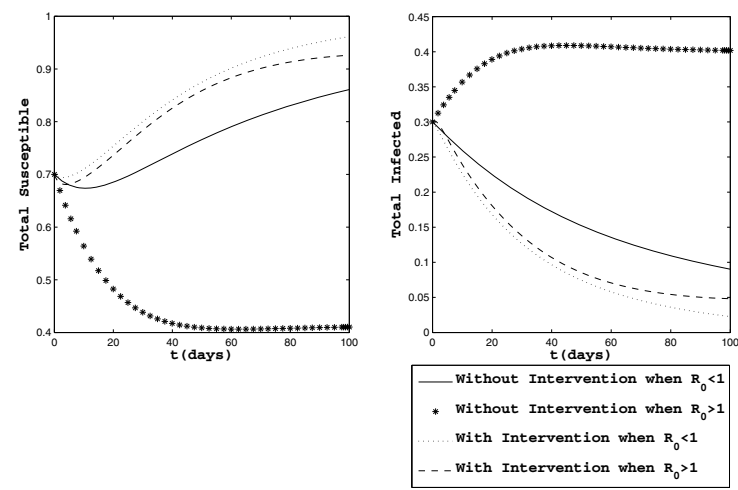

Fig. 11. Dynamics of total susceptible (left panel) and infected (right panel) populations with and without intervention for different magnitudes of $\mathcal{R}_{0}$.
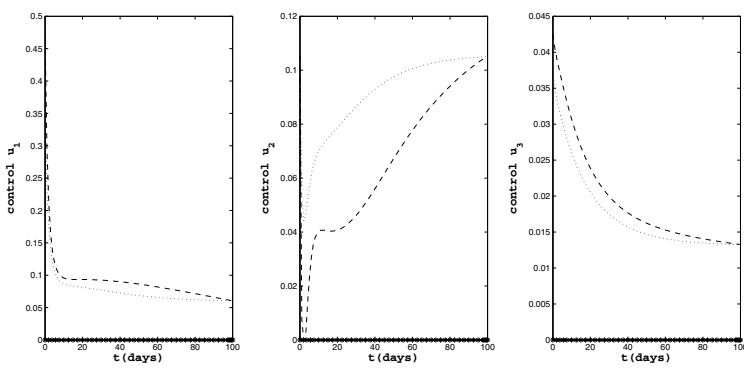

Fig. 12. Dynamics of control intervention for different magnitudes of $\mathcal{R}_{0}$. can accelerate the increase in the susceptible population for the scenario of $\mathcal{R}_{0}<1$, and push the dynamics of the susceptible population into a disease-free equilibrium for the case of $\mathcal{R}_{0}>1$, as shown in Fig. 11. The dynamics of the control variables for both the scenarios are almost the same. However, a greater effort should be applied in the government campaign when $\mathcal{R}_{0}>1$ since it needed $u_{2}$ almost twice as low as in the case of $\mathcal{R}_{0}<1$. As a result, we have a higher cost function value for the case of $\mathcal{R}_{0}>1$, which reaches 0.2483 and only 0.2117 when $\mathcal{R}_{0}<1$.

\subsubsection{Simulation with different budget limitations.} In this section, simulations were based on different preferences for the control strategy and conducted with different initial conditions (the endemic prevention and reduction scenarios). For this scenario, we assume that the government as a stage holder had limited preferences with regard to conducting an intervention to control the MERS disease. They had to choose between making an intervention using only medical masks $\left(u_{3}=0, u_{1}, u_{2}>\right.$ $0)$ or supportive care only $\left(u_{1}=u_{2}=0, u_{3}>0\right)$. This situation might arise when the government has a limited budget to control the spread of MERS.

Table 4. Table of numerical results for 4 different intervention strategies.

\begin{tabular}{|c|ccc|}
\hline Scenario & \multicolumn{3}{|c|}{ Endemic prevention } \\
& $s_{1}+s_{2}$ & $i_{1}+i_{2}$ & $\mathbf{J}$ \\
\hline \hline$u_{i}=0$ & 0.3302 & 0.4590 & 1.2465 \\
\hline$u_{1}, u_{2}>0, u_{3}=0$ & 0.9434 & 0.0392 & 0.0269 \\
\hline$u_{1}=u_{2}=0, u_{3}>0$ & 0.3627 & 0.4310 & 1.1366 \\
\hline$u_{i} \geq 0$ & 0.9425 & 0.0397 & 0.0288 \\
\hline \hline Scenario & \multicolumn{3}{|c|}{ Endemic reduction } \\
& $s_{1}+s_{2}$ & $i_{1}+i_{2}$ & $\mathbf{J}$ \\
\hline \hline$u_{i}=0$ & 0.3329 & 0.4546 & 1.9643 \\
\hline$u_{1}, u_{2}>0, u_{3}=0$ & 0.9211 & 0.0527 & 0.265 \\
\hline$u_{1}=u_{2}=0, u_{3}>0$ & 0.3658 & 0.4267 & 1.7988 \\
\hline$u_{i} \geq 0$ & 0.9205 & 0.0529 & 0.2585 \\
\hline
\end{tabular}

In both endemic prevention and reduction scenarios, an interesting finding is that the medical mask intervention only $\left(u_{1}, u_{2}>0\right)$ is the best option for reducing the number of infected humans as low as possible and increasing that of susceptible humans as high as possible 
(see Table 4 for details), rather than carrying out both interventions (medical mask and medical treatment, $u_{i}>$ $0, i=1,2,3)$. From the dynamics of infected individuals for endemic prevention and the reduction scenario with respect to time in Figs. 13 and 14, respectively, it can be seen that the medical mask intervention only and intervention using both medical masks and supportive care had only slightly different results. On the other hand, pushing intervention only through supportive care is the most ineffective strategy to be implemented. These results are a consequence of control trajectories, as shown in Fig. 15 for the endemic prevention scenario and in Fig. 16 for the endemic reduction scenario.

If we rely on the magnitude of the functional cost, from Table 4 also we can see that the medical mask intervention in endemic prevention is the cheapest strategy $(J=0.0269)$ to implement in the field, rather than including all interventions to be implemented $(J=$ 0.0288). Therefore, we could conclude that, instead of sharing the medical mask intervention budget with supportive care to infected people, it would be better to maximize the budget effort for medical mask intervention

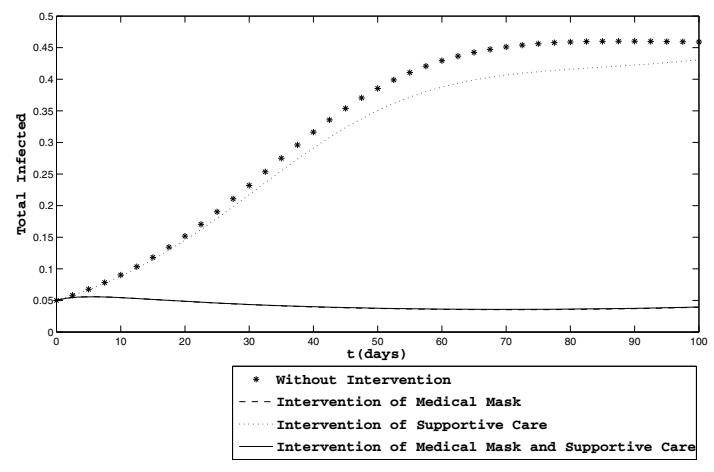

Fig. 13. Dynamics of infected individuals for the endemic prevention scenario with the preference of control strategies.

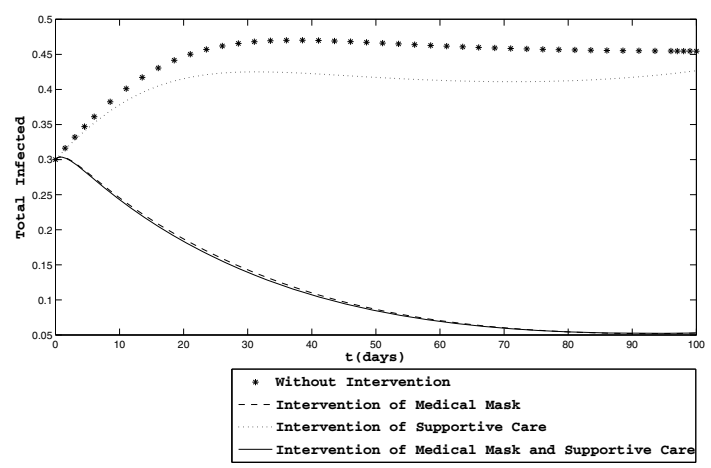

Fig. 14. Dynamics of infected individuals for the endemic reduction scenario with the preference of control strategies.
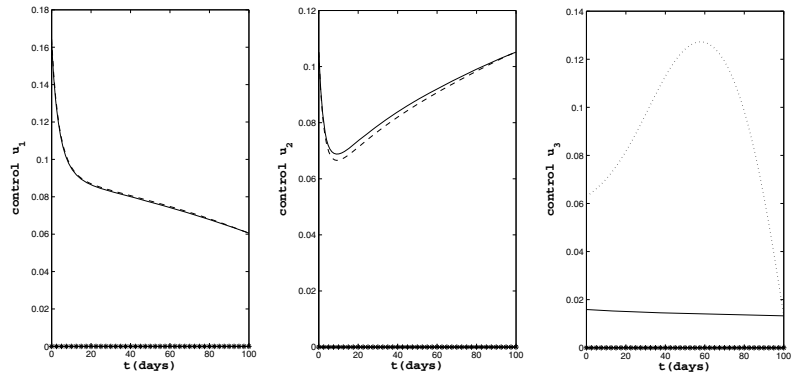

Fig. 15. Dynamics of $u_{i}(t)$ for various control strategies to produce the dynamics in Fig. 13
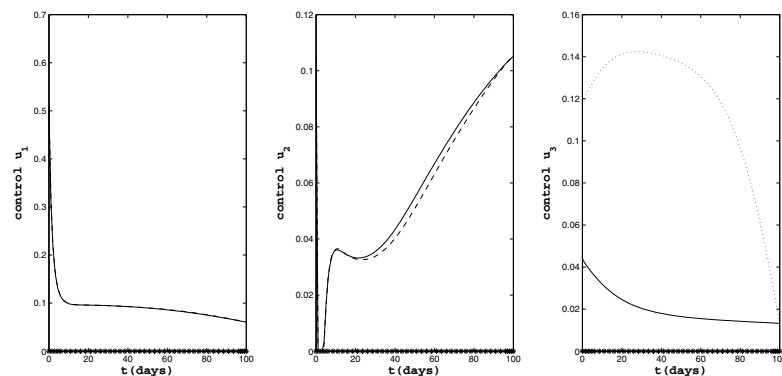

Fig. 16. Dynamics of $u_{i}(t)$ for various control strategies to produce the dynamics in Fig. 14

use and campaign about its importance only in the endemic prevention scenario.

In contrast to the endemic prevention scenario, in the endemic reduction case, although the medical mask intervention only is the best strategy to control MERS, it entails a higher cost, rather than integrating medical mask use and supportive care together (see Table 4 for details). Therefore, with a limited budget available to control MERS spread in the endemic reduction scenario, interventions with supportive care should be implemented to cure infected people and protect the rest of the population from the possibility of a new infection by using a medical mask.

\section{Conclusions}

In this article, we derived and analyzed a mathematical model for controlling the MERS disease with medical masks along with a campaign to promote the importance of this and of supportive care interventions. The problem is formulated as an optimal control one to minimize the number of infected people at a smaller cost.

Before the optimal control problem characterization, the autonomous system is analyzed to find all equilibrium points. We find the disease-free and endemic equilibrium points whose existence and local stability depend on the basic reproduction number $\left(\mathcal{R}_{0}\right)$. We learn that the 
endemic equilibrium point will always be positive for all categories if $\mathcal{R}_{0}>1$. We also find that the disease-free equilibrium will be locally asymptotic stable if and only if $\mathcal{R}_{0}<1$.

From the sensitivity analysis of the basic reproduction number with respect to all control variables, we find that medical mask intervention $\left(u_{1}\right.$ and $\left.u_{2}\right)$ is much more better for effecting a change in the basic reproduction number. The implication of this phenomenon is that, for effective eradication and control strategy for MERS, the focus should be on the medical mask intervention rather than the supportive care strategy.

To assess the impact of control variables for different scenarios, we conducted some simulations of the optimal control problem. According to the numerical results, an intervention should be conducted at the beginning of a MERS epidemic (endemic prevention scenario) to avoid high cost for interventions and achieve the lowest level of number of infected people. If there is a limited budget for interventions, concentrating on medical mask intervention for the endemic prevention scenario is the cheapest and best way to control the MERS spread. On the other hand, if an intervention is implemented in the middle of a MERS epidemic, medical mask intervention should be accompanied with the supportive care intervention.

From many studies (Muller et al., 2015), the camel is suspected to be the vector for spreading MERS under some circumstances. Therefore, a mathematical model involving the camel and constructing the model as a vector-borne disease model are important for the analysis. For the optimal control problem, intervention using a control function should be tried as a pulse control (Abboubakar et al., 2015) to find a more relevant intervention for practical purposes.

\section{Acknowledgment}

The authors thank all reviewers for their comments and suggestions that improved this article. This research is funded by the Indonesia Ministry of Research and Higher Education (Kemenristek DIKTI) with the PUPT research grant scheme 2016 (project ID: 1125/UN2.R12/HKP.05.00/2016).

\section{References}

Abboubakar, M., Kamgang, J. and Tieudjo, D. (2015). Backward bifurcation and control in transmission dynamics of arboviral diseases, Mathematical Biosciences 278(1): 100-129.

Al-Tawfiq, J., Smallwood, C., Arbuthnott, K., Malik, M.S., Barbeschi, M. and Memish, Z. (2012). Emerging respiratory and novel coronavirus 2012 infections and mass gatherings, East Mediterr Health Journal 19(1): 48-54.
Aldila, D., Nuraini, N. and Soewono, E. (2014). Optimal control problem of preventing of swine flu disease transmission, Applied Mathematical Science 8(71): 3501-3512.

Aldila, D., Soewono, E. and Nuraini, N. (2012). On the analysis of effectiveness in mass application of mosquito repellent for dengue disease prevention, AIP Conference Proceedings 1450(1): 103-109.

Assiri, A., McGeer, A., Perl, T., Price, C., Al Rabeaah, A. and Cummings, D. (2013). Hospital outbreak of Middle East respiratory syndrome coronavirus, The New England Journal of Medicine 369(5): 407-416.

Cauchemez, S., Fraser, C., Van Kerkhove, M., Donnelly, C., Riley, S. and Rambaut, A. (2014). Middle East respiratory syndrome coronavirus: Quantification of the extent of the epidemic, surveillance biases, and transmissibility, Lancet Infectious Diseases 14(1): 5056.

Chowell, G., Blumberg, S., Simonsen, L., Miller, M. and Viboud, C. (2014). Synthesizing data and models for the spread of MERS-CoV, 2013: Key role of index cases and hospital transmission, Epidemics 9(1): 40-51.

Diekmann, O. and Heesterbeek, J. (2000). Mathematical Epidemiology of Infectious Diseases, Model Building, Analysis and Interpretation, John Wiley \& Son, Chichester.

Diekmann, O., Heesterbeek, J. and Metz, J. (1990). On the definition and the computation of the basic reproduction ratio of $\mathcal{R}_{0}$ in models of infectious disease in heterogeneous populations, Journal of Mathematical Biology 28(4): 365-382.

Diekmann, O., Heesterbeek, J. and Roberts, M. (2010). The construction of next-generation matrices for compartmental epidemic models, Journal of The Royal Society Interface 7(47): 873-885.

Ejima, K., Aihara, K. and Nishiura, H. (2014). Probabilistic differential diagnosis of Middle East respiratory syndrome (MERS) using the time from immigration to illness onset among imported cases, Journal of Theoretical Biology 346(1).

Gautret, P. (2013). Middle East respiratory syndrome (MERS) coronavirus: What travel health advice should be given to Hajj pilgrims?, Travel Medicine and Infectious Disease 11(5): 263-265.

Gerberry, D. (2016). Practical aspects of backward bifurcation in a mathematical model for tuberculosis, Journal of Theoretical Biology 388(1): 15-36.

Haagmans, B., Al Dhahiry, S., Reusken, C., Raj, V. and Galiano, M. (2014). Middle East respiratory syndrome coronavirus in dromedary camels: An outbreak investigation, Lancet Infectious Diseases 14(2): 140-145.

Malik, T.M., Alsaleh, A.A., Gumel, A.B. and Safi, M.A. (2015). Optimal strategies for controlling the MERS coronavirus during a mass gathering, Global Journal of Pure and Applied Mathematics 11(6): 4831-4865.

Muller, M., Meyer, B., Corman, V., Al-Masri, M., Turkestani, A. and Ritz, D. (2015). Presence of Middle East respiratory syndrome coronavirus antibodies in Saudi Arabia: A nationwide, cross-sectional, serological study, Lancet Infectious Diseases 15(5): 559-564. 
Novkaniza, F., Ivana and Aldila, D. (2016). Controlling influenza disease: Comparison between discrete time Markov chain and deterministic model, AIP Conference Proceedings 1723(1): 030015-10, DOI: 10.1063/1.4945073.

Obaid, H.A., Ouifki, R. and Patidar, K.C. (2013). An unconditionally stable nonstandard finite difference method applied to a mathematical model of HIV infection, International Journal of Applied Mathematics and Computer Science 23(2): 357-372, DOI: 10.2478/amcs-2013-0027.

Okuonghae, D. (2013). A mathematical model of tuberculosis transmission with heterogeneity in disease susceptibility and progression under a treatment regime for infectious cases, Applied Mathematical Modelling 37(10-11): 6786-6808.

Omrani, A., Abdul-Mutin, M., Haddad, Q., Al-Nakhli, D., Memish, Z. and Albarrak, A. (2013). A family cluster of Middle East respiratory syndrome coronavirus infectious related to a likely unrecognized asymptomatic or mild case, International Journal of Infectious Disease 17(9): 668-672.

Paez Chavez, J., Gotz, T., Siegmund, S. and Wijaya, K. (2017). An SIR-Dengue transmission model with seasonal effects and impulsive control, Mathematical Biosciences 289(2): 29-39.

Pattnaik, S., Bakwad, K., Sohi, B., Ratho, R. and Devi, S. (2013). Swine influenza models based optimization (SIMBO), Applied Soft Computing 13(1): 628-653.

Poletto, C., Pelat, C., Levy-Bruhl, D., Yazdanpanah, Y., Boelle, P.-Y. and Colizza, V. (2014). Assessment of the Middle East respiratory syndrome coronavirus (MERS-COV) epidemic in the Middle East and risk of international spread using a novel maximum likelihood analysis approach, Eurosurveillance 19(23): 20824.

Reusken, C.B.E.M., Haagmans, B.L., Muller, M.A., Gutierrez, C., Godeke, G.J., Meyer, B., Muth, D., Raj, V.S., Smits-De Vries, L., Corman, V.M., Drexler, J.-F., Smits, S.L., El Tahir, Y.E., De Sousa, R., van Beek, J., Nowotny, N., van Maanen, K., Hidalgo-Hermoso, E., Bosch, B.J., Rottier, P., Osterhaus, A., Gortazar-Schmidt, C., Drosten, C. and Koopmans, M.P.G. (2013). Middle East respiratory syndrome coronavirus neutralising serum antibodies in dromedary camels: A comparative serological study, Lancet Infectious Diseases 13(10): 859-866.

Saha, S. and Roy, P.K. (2017). A comparative study between two systems with and without awareness in controlling HIV/AIDS, International Journal of Applied Mathematics and Computer Science 27(2): 337-350, DOI: 10.1515/amcs-2017-0024.

WHO (2013). Revised interim case definition for reporting to WHO-Middle East respiratory syndrome coronavirus (MERS-CoV), www.who.int/csr/disease/coro navirus_infections/case_definition_03_ $07 \_2014 / \mathrm{en} /$.

WHO (2016). Middle East respiratory syndrome coronavirus (MERS-CoV), www.who.int/mediacentre/fact sheets/mers-cov/en
Xia, Z.-Q., Zhang, J., Xue, Y.-K., Sun, G.-Q. and Jin, Z. (2015). Modeling the transmission of Middle East respirator syndrome corona virus in the Republic of Korea, PLOS ONE 10(12): e0144778.

$\mathrm{Xu}, \mathrm{Z}$. and Ai, C. (2016). Traveling waves in a diffusive influenza epidemic model with vaccination, Applied Mathematical Modelling 40(15-16): 7265-7280.

Zaki, A., van Boheemen, S., Bestebroer, T., Osterhaus, A. and Fouchier, R. (2012). Isolation of a novel coronavirus from a man with pneumonia in Saudi Arabia, The New England Journal of Medicine 367(19): 1814-1820.

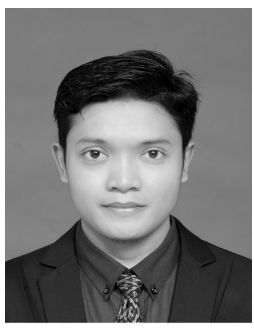

Dipo Aldila graduated from Tadulako University in 2009. He received his Master's and PhD degrees from Institut Teknologi Bandung in 2011 and 2014, respectively. He is currently a lecturer in the Department of Mathematics, Universitas Indonesia. His present research interests are in mathematical modeling and optimal control, especially the disease spread model.

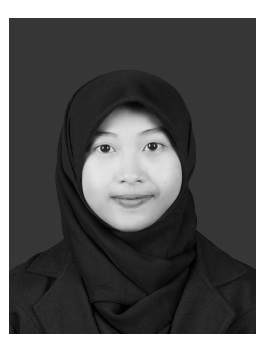

Herningtyas Padma received her BSc in 2015 from the Department of Mathematics, Universitas Indonesia, under the supervision of Dipo Aldila and Hengki Tasman. Her research interests are in disease spread modeling with ordinary differential equations.

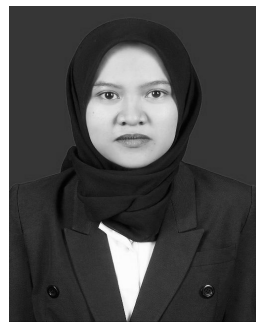

Khusnul Khotimah received her BSc in 2015 from the Department of Mathematics, Universitas Indonesia, under the supervision of Dipo Aldila and Bevina D. Handari. Her research interests are in application of optimal control to disease modeling.

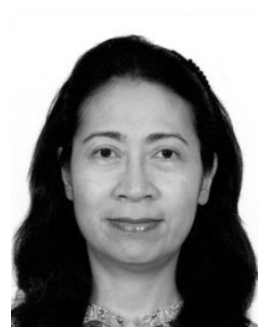

Bevina Desjwiandra obtained her $\mathrm{PhD}$ in mathematics from the University of Queensland in 2003. In her early academic years she was at Universitas Indonesia (1986) and Michigan State University (1992). She has been a lecturer at the Department of Mathematics, Faculty of Mathematics and Natural Sciences, Universitas Indonesia, since 1987. Her current research interests are in computational finance, heuristic optimization and biomathematics. 


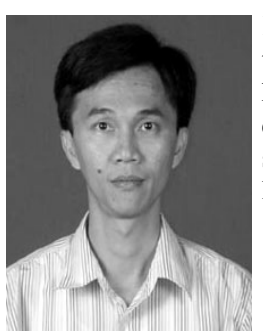

Hengki Tasman received his $\mathrm{PhD}$ from Institut Teknologi Bandung in 2010. Since 2002, he has been a lecturer in the Department of Mathematics, Universitas Indonesia. His current research interests are in biomathematics and population dynamics.

Received: 7 November 2016

Revised: 18 April 2017

Re-revised: 7 September 2017

Accepted: 10 October 2017 